\title{
MigRACIÓN, FUERZA DE TRABAJO Y FAMILIA, ELEMENTOS EN LA DEFINICIÓN DEL ESPACIO TRANSFRONTERIZO MÉXICO-GUATEMALA ${ }^{1}$
}

\section{Migration, Workforce and FAMily, Elements to Define the Transborder Space Mexico-Guatemala}

\section{Jéssica N. Nájera Aguirre ${ }^{2}$}

Resumen: A lo largo del siglo XX los estudios acerca de la movilidad laboral transfronteriza entre México y Guatemala han mostrado la incorporación de nuevos actores, vínculos e instituciones y particularmente en la histórica región del Soconusco, en el estado de Chiapas. Este trabajo tiene el propósito de incorporar al estudio de los procesos transfronterizos la dimensión familiar, como un elemento que contribuye a la configuración de un espacio transfronterizo más allá de considerar únicamente la dimensión laboral. Desde una perspectiva sociodemográfica y a través de una aproximación metodológica mixta, se muestra la existencia de dinámicas familiares transfronterizas producto del trabajo migratorio de commuting, temporal o casi permanente de los guatemaltecos al Soconusco; es así que lo familiar, social y laboral dan forma a la reproducción social y cotidiana de las poblaciones de ambos lados de la frontera.

Palabras clave: trabajadores migratorios, hogares, frontera, guatemaltecos, Chiapas.

\footnotetext{
${ }^{1}$ Nota del editor: se respeta íntegramente el texto final que entregó la autora, con los dictámenes académicos atendidos.

${ }^{2}$ Centro de Estudios Demográficos, Urbanos y Ambientales (CEDUA). El Colegio de México. Correo electrónico: jnajera@colmex.mx

Fecha de recepción: 2701 17; Fecha de aceptación: 110517.
}

(cc) EY-NC-ND Páginas 119-150. 
Abstract: Throughout the twentieth century studies on cross-border labor mobility between Mexico and Guatemala have shown the incorporation of new actors, links and institutions, particularly in the historic Soconusco region in the state of Chiapas. This paper aims to incorporate the family dimension to the study of cross-border processes as an element that contributes to the configuration of a crossborder space beyond considering only the labor dimension. From a sociodemographic perspective and through a mixed methodological approach, the existence of transborder family dynamics is demonstrated by the migratory work of commuting, temporary or almost permanent, of the Guatemalans to Soconusco; so the family and work shape the social and daily reproduction of populations on both sides of the border.

Keywords: migrant workers, households, border, guatemalans,

Chiapas.

\section{A manera de introducción: el contexto transfronterizo México-Guatemala}

La frontera sur de México con el vecino país de Guatemala ha sido históricamente un espacio de vinculación laboral, cultural y social. Si bien, en la región fronteriza del lado mexicano se encuentran los estados de Chiapas, Tabasco y Campeche, y del lado guatemalteco, los departamentos de San Marcos, Retalhuleu, Quetzaltenango, Suchitepéquez, Sololá y Totonicapán -pertenecientes a la región Sur occidente de Guatemala; la mayor dinámica transfronteriza en esta región se ha concentrado históricamente en la demarcada por Chiapas y San Marcos (Palma, 2003), por ser un espacio de relaciones históricas de conquista, comercio y demanda de trabajadores agrícolas y el punto geográfico en el que se conecta la infraestructura carretera que llega desde Centroamérica a México.

Esta parte de la frontera sur de México es un espacio en el que ocurren esencialmente tres dinámicas de movilidad y migración simultáneas, cuya dirección poblacional va de sur a norte, es decir, guatemaltecos que cruzan a Chiapas. La primera es el gran intercambio poblacional, 
comercial y laboral transfronterizo guatemalteco al Soconusco; el segundo es el tránsito de transmigrantes, provenientes principalmente de Guatemala, Honduras y El Salvador, cuyo destino final es Estados Unidos de América; y finalmente, la inmigración de guatemaltecos que constantemente llegan a residir a Chiapas. En este espacio fronterizo resaltan dos características que permiten comprender las relaciones de movilidad, migración y fuerza de trabajo en esta zona fronteriza: el tipo de frontera entre ambos países, y las características socioeconómicas de la región transfronteriza.

\section{La frontera como espacio de regulación migratoria}

La frontera entendida como la línea de separación entre dos países es reconocida por las poblaciones locales de cada territorio nacional a través de dos elementos: el límite fronterizo y el tipo de regulación y control migratorio existente. La política migratoria del estado mexicano respecto a su frontera sur ha mantenido históricamente una separación fronteriza sin muros o vallas, por lo que queda enmarcada por señalamientos naturales, como el Río Suchiate, y por señalamientos materiales, como pequeños montículos de cemento entre el espeso contexto de selva que hay entre México y Guatemala. Sin muros ni vallas que imposibiliten el tránsito de poblaciones de un lado de esta frontera, la idea de que existe una "frontera abierta" se ha mantenido. Si bien existen siete puntos fronterizos formales de paso entre estos países, ${ }^{3}$ existen cientos de puntos fronterizos informales de paso a lo largo de la línea fronteriza, por los cuales cruza población guatemalteca que se dirige a visitar, hacer compras y trabajar en Chiapas.

La delimitación de los territorios nacionales es uno de los insumos para la definición de las políticas de regulación y control migratorio nacional. En la frontera sur de México existen una serie de regulaciones migratorias cuyo propósito ha sido permitir la entrada ordenada y regulada de población guatemalteca al territorio mexicano, la cual puede ser con propósitos laborales y no laborales, y de manera temporal; por lo que puede considerarse que existe una política migratoria mexicana

\footnotetext{
${ }^{3}$ Ciudad Hidalgo-Tecún Umán, Suchiate II, Talismán-El Carmen, Unión Juárez-Toquián Grande, Ciudad Cuauhtémoc (Las Champas) -La Mesilla, Carmen Xhan-Gracias a Dios, Frontera Corozal-Bethel.
} 
privilegiada con Guatemala respecto a las movilidades transfronterizas. Históricamente la presencia de trabajadores guatemaltecos en Chiapas ha sido permitida aunque cada vez más regulada con el paso del tiempo. Desde 1997 los guatemaltecos de los departamentos fronterizos con México de San Marcos, Huehuetenango y Quetzaltenango, ${ }^{4}$ podían obtener un documento migratorio para cruzar al estado de Chiapas a trabajar en el sector agrícola. La Forma Migratoria de Visitante Agrícola (FMVA), emitida por el Instituto Nacional de Migración (INM), fue el primer documento personalizado que permitió la movilidad laboral transfronteriza de trabajadores guatemaltecos a las fincas cafetaleras de la región del Soconusco.

En 2005 a partir de la Propuesta de Política Migratoria Integral del INM y con la emisión de la Forma Migratoria de Trabajador Fronterizo (FMTF) ${ }^{5}$, se ampliaron las posibilidades de trabajo en Chiapas al incorporar a los trabajadores no agrícolas y a aquéllos procedentes de departamentos no fronterizos. Más recientemente, con la Ley de Migración de 2011, la movilidad laboral transfronteriza de guatemaltecos a México es permitida para todos los guatemaltecos que deseen desempeñar cualquier oficio o profesión lícita en las entidades del sur de México, independientemente del departamento procedencia, obteniendo una Tarjeta de Visitante Trabajador Fronterizo (TVTF). ${ }^{6}$

Si bien, las posibilidades de regulación y control migratorio respecto a los trabajadores migratorios guatemaltecos en la frontera sur de México se han ampliado en el tiempo, en la cotidianidad aún existe una incipiente documentación migratoria laboral, que dificulta conocer la magnitud de este flujo de trabajadores migratorios en el territorio mexicano. Las razones de tal situación suelen ser tres: la dificultad de realizar el trámite migratorio, ya que debe participar el empleador; la

\footnotetext{
${ }^{4}$ San Marcos, Huehuetenango y Quetzaltenango.

${ }^{5}$ En marzo de 2008 se aprobó la Forma Migratoria de Trabajador Fronterizo (FMTF), publicado en el Diario Oficial de la Federación, Tomo DCLIV, No.9, México, D.F. 12 de marzo de 2008. Esta forma migratoria fue válida entre el 2008 y el 2012.

${ }^{6}$ La Tarjeta de Visitante Trabajador Fronterizo (TVTF) se creó con base en la Ley de Migración 2011 y su correspondiente Reglamento en el ańo 2012, publicado en el Diario Oficial de la Federación, 8 de noviembre de 2012, Artículos 75, 76 y 77, México, D.F. La TVTF permite a los guatemaltecos y beliceños, de 16 años o más, desempeñar alguna actividad laboral (agrícola y no agrícola) en los estados del sur de México -Chiapas, Tabasco, Campeche y Quintana Roo.
} 
idea por parte de trabajadores y empleadores de "no necesitar" un documento que les autorice trabajar, especialmente cuando tienen acceso a uno que les avala el cruce y la estancia temporal en México - como es la Tarjeta de Visitante Regional (TVR) que les permite entrar permanecer en México por varios días; ${ }^{7}$ y finalmente, las posibilidades que surgen de una "frontera abierta", de entrar, permanecer y trabajar en México, sin contar con un documento migratorio que avale su estancia laboral, pero además la existencia de acuerdos informales locales para permitir las movilidades transfronterizas.

Sin embargo, en el actual contexto migratorio mexicano, los trabajadores guatemaltecos transfronterizos presentan una mayor tendencia a documentar su estancia en Chiapas con el propósito de evitar ser confundidos con migrantes en tránsito y ser devueltos a Guatemala. Este hecho permite mostrar que los trabajadores transfronterizos tienen una posición privilegiada para la entrada y estancia laboral en territorio mexicano respecto a otras poblaciones migrantes, que incluso también trabajan en el mercado laboral chiapaneco como hondureños, salvadoreños y nicaragüenses, pero que no son beneficiarios de la documentación migratoria laboral existente en la frontera sur de México.

En resumen, la frontera México-Guatemala es un espacio que por el tipo de límite fronterizo, la regulación migratoria fronteriza existente y las relaciones transfronterizas de tolerancia a las movilidades locales, permite una vida y dinámica transfronteriza y una alta movilidad de fuerza de trabajo migrante de origen guatemalteco a los estados de la frontera sur de México y en particular al Soconusco chiapaneco.

\section{Características socioeconómicas del espacio transfronterizo}

Como señala Arriaga (2012) "la frontera es un espacio geográfico complejo en donde ocurren fenómenos sociales de naturaleza diversa y que tienen incidencia en el desarrollo de los Estados"; en

\footnotetext{
${ }^{7}$ La Tarjeta de Visitante Regional (TVR) se creó con base en la Ley de Migración 2011 y su correspondiente Reglamento en el ańo 2012, de acuerdo al Diario Oficial de la Federación, 8 de noviembre de 2012, Artículo 73. La TVR es un documento migratorio que les permite a los guatemaltecos y beliceños, cruzar a ciudades fronterizas de México, para ir de visita o de compras, hasta por un máximo de tres días, con posibilidades de múltiples cruces. Se tramita en las oficinas del INM ubicadas en los Puntos de internación a México y tiene una vigencia de cinco años a partir de su expedición.
} 
este sentido, el espacio transfronterizo México-Guatemala no es la excepción. Uno de los procesos sociales más relevantes e históricos es la dinámica laboral que ocurre, tanto de manera individual como conjunta entre las poblaciones de ambos lados de la línea fronteriza. La presencia de trabajadores guatemaltecos en Chiapas está fundamentada principalmente en la constante demanda de fuerza de trabajo en el mercado laboral de la región del Soconusco y la consecuente oferta de trabajadores provenientes de Guatemala y en particular de la región del suroccidente. Esta dinámica laboral transfronteriza vincula y beneficia, aunque no de manera igual, a la economía, el desarrollo económico y las poblaciones de México y Guatemala.

El espacio geográfico en conjunto que ocupa la región del Soconusco y del Sur-Occidente de Guatemala (Mapa 1) se caracteriza por la similitud y la continuidad socioeconómica en dos sentidos: primero, las similitudes climáticas y socioproductivas que refieren a formas similares de reproducción de las sociedades de ambos lados de la línea fronteriza, en particular la producción agrícola para la subsistencia familiar — autoconsumo - o como un medio para obtener recursos - negocio o empresa - en productos similares como el café, maíz, banano y palma africana. En segundo lugar, existen similitudes económicas, culturales y lingüísticas entre las poblaciones chiapanecas y guatemaltecas cercanas a la línea fronteriza, en su mayoría son poblaciones campesinas, indígenas, hablantes de español y lenguas indígenas, con altas tasas de pobreza y en algunos casos en subsistencia alimentaria (PNUD, 2011).

Respecto al contexto económico-laboral en las zonas de frontera de ambos lados de México-Guatemala existen desigualdades de desarrollo económico local, que diferencia el espacio chiapaneco del guatemalteco. El lado mexicano se ha configurado como un espacio laboral de alta demanda de fuerza de trabajo, tanto en la economía rural como en la economía urbana, particularmente como resultado de una economía de agroexportación y del crecimiento y dinámica de la ciudad de Tapachula, principal centro urbano de la región del Soconusco. Por su parte, en el lado guatemalteco existe un desarrollo económico local comparativamente menor que el mexicano, a pesar de que también existen espacios laborales destinados a la producción agrícola de exportación como en 
Chiapas. En los departamentos guatemaltecos fronterizos existen pocos centros urbanos enfocados en dinámicas de consumo local y abastecimiento de bienes y servicios básicos para la población fronteriza, como las localidades de Tecún Umán, Malacatán y San Marcos, pero ninguno del tamaño y dinamismo que Tapachula (Chiapas), por ello este territorio mexicano es considerado el centro urbano más importante de la región transfronterizo.

Mapa 1. Región transfronteriza México-Guatemala.

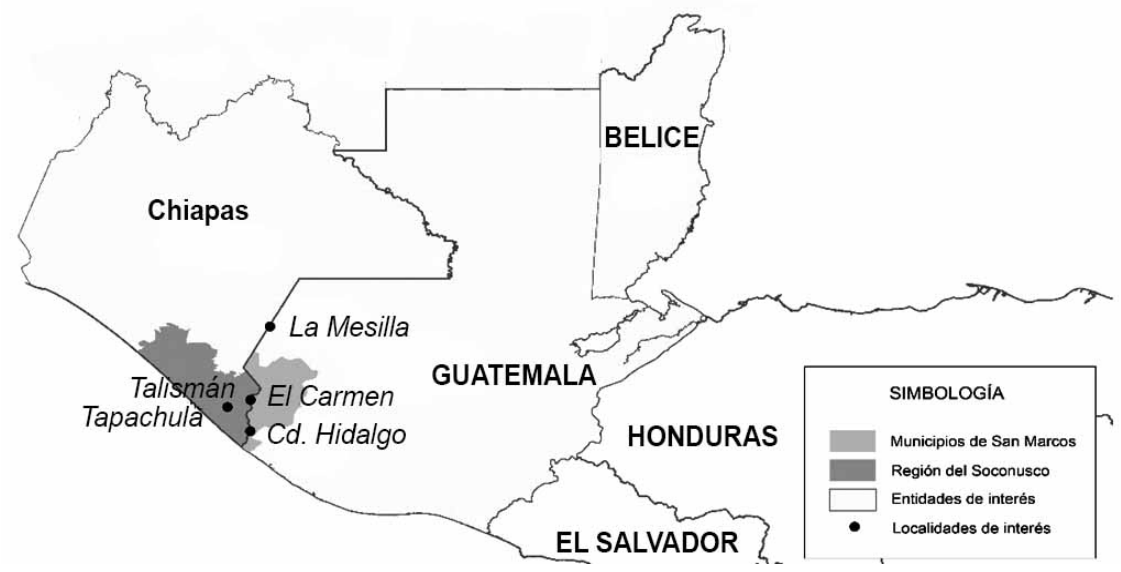

Fuente: Elaboración propia, Nájera (2017)

Las similitudes económico-productivas mexicanas y guatemaltecas propician una especialización laboral semejante de ambos lados de la línea fronteriza, identificando por ejemplo una región en la que se produce café denominada como la "zona fría", y una región bananera y dedicado a la producción de otros frutos tropicales conocida como la "zona caliente" ubicada en los límites del Océano Pacífico; ambas zonas se extienden desde Guatemala a Chiapas. Esta característica climáticaproductiva se traduce en una ventaja para la dinámica económica de la región transfronteriza y en especial para el mercado laboral chiapaneco, ya que propicia un emparejamiento entre la demanda y oferta de fuerza de trabajo a partir de la cualificación y experiencia laboral en la producción agrícola. La demanda de trabajadores agrícolas en el Soconusco es permanente y por temporadas; estas últimas ocurren 
especialmente en la época de cosecha de café o de corte de caña; mientras que las contrataciones permanentes, se observan en la producción de banano, mango, papaya, limón, etc. o incluso en el café y la caña para mantener un stock de trabajadores (varias de estos trabajadores son analizados en Rivera, 2014).

En el caso del mercado laboral urbano del Soconusco se ha mostrado que los trabajadores guatemaltecos laboran en actividades como el trabajo doméstico, el trabajo en la construcción, los servicios, el comercio y la venta ambulante, principalmente en ciudades como Tapachula. Por otro lado, si bien la fuerza de trabajo guatemalteca es demandada en restaurantes, tiendas, pequeñas empresas, casashabitación, por mencionar algunos espacios laborales; también hay trabajadores por cuenta propia que desempeñan sus actividades para negocios, tiendas o laboran en la calle, ofreciendo sus servicios - cargadores y boleros - o vendiendo productos como dulces, frutas, frituras, verduras, medicinas, ropa usada, etc.

Los principales vínculos entre demanda y oferta de fuerza de trabajo migrante son los "contratistas" o "enganchadores" de origen guatemalteco contratados por los empleadores y las redes familiares, de vecinos, amigos y paisanos, que transmiten las necesidades de mano de obra en México y las posibilidades de trabajo cuenta propia en el mercado laboral mexicano.

En general, las características migratorias y laborales de la región transfronteriza México-Guatemala muestran continuidades y cambios a lo largo del tiempo, es decir, han permanecido las necesidades de mano de obra en la región del Soconusco y la disponibilidad y movilidad de trabajadores guatemaltecos transfronterizos; ${ }^{8}$ mientras que han variado los nichos de trabajo y las condiciones migratorias y laborales de los trabajadores. En este contexto para este trabajo resultó relevante preguntarse acerca de la vida cotidiana de los trabajadores y sus familiares en este espacio transfronterizo, como un acercamiento a las formas de reproducción social y generacional de la fuerza de trabajo migrante guatemalteca en el Soconusco.

\footnotetext{
${ }^{8}$ Para una referencia histórica de la presencia de trabajadores guatemaltecos en Chiapas ver Zebadúa (1999).
} 


\section{El estudio de las familias a través del trabajo migratorio y la reproducción social cotidiana}

La familia ha sido definida de diferentes maneras dependiendo de la disciplina desde la cual se estudia; en el caso de la sociodemografía, la familia es el conjunto de individuos vinculados entre sí por lazos de parentesco - a partir de una relación consanguínea, adopción, matrimonio o unión-, que viven en un mismo espacio residencial en el que comparten la domesticidad y la vida económica. La sociología agrega a la visión sociodemográfica de familia tres elementos relevantes: en la familia se llevan a cabo funciones sociales particulares como la reproducción social y cotidiana; existe intimidad, solidaridad y duración de la relación entre sus integrantes; y es una institución que representa un conjunto de normas, vínculos y roles para cada uno de sus integrantes los cuales son definidos culturalmente (Godoy y Lira, 1973).

Con estos referentes, el interés del estudio de las familias guatemaltecas en contextos transfronterizos pone interés en aspectos como: quiénes conforman el grupo social, qué tipo de parentesco existe entre ellos, quiénes corresiden de manera habitual, cómo se organizan para llevar a cabo la reproducción social y cotidiana, qué tarea y roles le corresponde o asume cada integrante en la familia y cuáles son las prácticas cotidianas para mantener dicha institución social. Estos intereses permitirán tener de manera general, la estructura y la dinámica de dichas familias.

Un primer reto en el estudio sobre las familias es distinguir, conceptualmente y con propósitos metodológicos, entre los conceptos de familia y hogar. Aunque ambos términos comparten el hecho de considerarse espacios en los que sus miembros llevan a cabo las actividades necesarias para su reproducción cotidiana, en la familia se requiere que sus integrantes estén vinculados por relaciones de parentesco, mientras que en el hogar sus integrantes pueden o no estarlo, es decir, pueden o no ser familia (Salles, 1991; Oliveira, 1989; Oliveira y Salles, 1989; entre otros). Por otra parte, en las familias todos sus integrantes pueden o no residir de manera habitual en el mismo espacio, y seguir siendo familia ya que son parte de la reproducción cotidiana y social de dicha unidad (Acosta, 2003; Salvia, 1995; entre otros). 
En la investigación sociodemográfica, la dificultad de utilizar conceptos como familia a través de encuestas y censos de población, propició la creación de conceptos alternativos, tal es el caso del concepto "hogar familiar", definido por Arriagada (2007), como un espacio en el que residen de manera habitual personas vinculadas por relaciones de parentesco y que comparten la reproducción cotidiana de sus integrantes. En contraparte se encuentra el "hogar no familiar" que refiere al grupo de personas que no está vinculados por relaciones de parentesco, aunque compartan tareas necesarias para mantener dicho espacio. En el caso de esta investigación, aunque el interés son las familias de los guatemaltecos que laboran en el Soconusco, su estudio se lleva a cabo a partir diversas aproximaciones metodológicas como son las movilidades laborales de las y los trabajadores entre Guatemala y México, y a partir de ellos la reconstrucción de las familias transfronterizas que a pesar de no vivir necesariamente en un mismo espacio residencial, comparten la domesticidad y la vida familiar.

\section{Las familias en contextos de migración}

El estudio de las familias en situaciones y contextos particulares, como es el caso de la migración y los espacios fronterizos, ha sido un tema de interés entre países de alto intercambio poblacional, como México y Estados Unidos. Los principales retos de estudiar a las familias en estos contextos de migración y movilidad transfronteriza es que dicha unidad de análisis se ve afectada en uno de sus determinismos básicos, que es la corresidencia de sus integrantes, debido a la salida de uno o varios de sus miembros hacia otro país de manera temporal o casi permanente. Diversos estudios han mostrado que las familias en contextos de migración son un ejemplo de que dicha institución social puede preservarse aunque sus integrantes residan en espacios distintos — unos en el país de origen y otros en el país de destino- y que pueden mantener vínculos y responsabilidades para la mantener la reproducción cotidiana de la misma. Ejemplo de esto último son las denominadas familias transnacionales, las cuales alejadas de cumplir con el requisito de la convivencia cotidiana bajo el mismo techo, basan su pertenencia a dicho grupo social en los lazos de parentesco y las 
relaciones de intercambio recíproco y lazos de solidaridad entre los que están en un país y en otro (Pedone, 2006; Woo, 2006). Es así que las familias pueden mantenerse como grupo social a través de los vínculos continuos entre ellos a pesar de la distancia y de la ubicación geográfica de sus integrantes.

Por su parte, las familias transfronterizas pertenecen a situaciones de movilidad y migración entre países vecinos. En estos casos, a pesar de la movilidad de población de un lado a otro de la línea fronteriza por motivos diversos, la familia suele mantener un lugar de residencia habitual en un lado de la frontera (Acuña, 1980; Alegría, 1989). Sin embargo, como ha mostrado Fauné (1996), este tipo de familias crean en un nuevo espacio residencial que sobrepasa el estricto espacio fijo en el que residen todos los miembros de manera permanente, y emergen nuevos lazos y lealtades entre ellos. En las familias transnacionales y transfronterizas, al menos uno de sus miembros está ausente del hogar, de manera temporal o permanente a otro país y mantienen una división y organización intrafamiliar básica para la reproducción cotidiana de la familia que incluye al o los ausentes.

\section{Reproducción social de las familias en situaciones de movilidad}

y migración internacional

Las familias con base en su estructura se organizan para llevar a cabo su reproducción social y cotidiana. Este trabajo considera la propuesta sociodemográfica de García y Oliveira (2011), respecto a comprender la dinámica interna de las familias, a partir desde dos ejes: la división del trabajo intrafamiliar y las formas de convivencia familiar. De acuerdo a las autoras, las familias se organizan internamente para realizar tres tareas esenciales en el hogar: el trabajo doméstico, el trabajo de cuidados y el trabajo extradoméstico — que permite la obtención de bienes y servicios necesarios para la manutención de los integrantes, vía trabajo remunerado o no remunerado como la producción agrícola para el autoconsumo. Con base en ello, un grupo social puede considerarse una familia cuando sus integrantes, emparentados entre sí, son parte de la división de tareas necesarias para mantener su reproducción social y cotidiana. 
En un "modelo tradicional de familia", el trabajo remunerado suele estar a cargo del varón jefe de hogar, quien es el principal proveedor económico; mientras que el trabajo doméstico y el trabajo de cuidados están a cargo de la esposa o cónyuge de dicho jefe. Es así que la división del trabajo intrafamiliar queda mediada por los roles y responsabilidades tradicionalmente asignados a partir del género y al que pertenecen. Por su parte García y Oliveira (2011) identifican tres aspectos esenciales para dar cuenta de las formas de convivencia familiar: las relaciones de género entre los cónyuges, a partir de la toma de decisiones, las formas de control conyugal y la violencia doméstica.

En las familias en situaciones de movilidad y migración internacional, una distribución interna "tradicional" de las tareas y responsabilidades de sus integrantes es una de las razones por la cual los varones jefes de hogar se convierten en migrantes internacionales al considerar la movilidad internacional como una alternativa de trabajo remunerado. Sin embargo, en las últimas décadas, las cónyuges también han abandonado la corresidencia habitual con sus hijos para compartir la proveeduría familiar, lo que ha implicado la falta de presencia para llevar a cabo su papel como responsables de las labores de la casa y del cuidado de los hijos.

Para el presente trabajo, la familia se ubica como la unidad de observación para analizar la relación entre la fuerza de trabajo migrante transfronteriza y la dinámica familiar en la región México-Guatemala, en un entorno de permisividad para la movilidad internacional fronteriza por razones de trabajo y un mercado laboral chiapaneco con demanda de fuerza de trabajo migrante.

Se implementó un acercamiento metodológico mixto para analizar la relación migración, fuerza de trabajo y familia, en este contexto transfronterizo. En la primera parte, con base en la Encuesta sobre Migración en la Frontera Sur de México (EMIF SUR) ${ }^{9}$ 2015, se presenta un panorama de la fuerza de trabajo migrante guatemalteca que participa en el mercado laboral chiapaneco, a través de los desplazamientos que éstos realizan por la zona fronteriza del Soconusco. Dicho panorama tiene como propósito mostrar las características

\footnotetext{
${ }^{9}$ Para ver la metodología de la Emif Sur ver Colef et al. (2011).
} 
geográficas transfronterizas —origen/destino_- y las posibilidades y espacios del trabajo migratorio desde una perspectiva familiar del trabajador que se desplaza a través de la frontera.

En la segunda parte, con base en entrevistas realizadas a trabajadores migratorios guatemaltecos que laboran en el Soconusco, se da cuenta de la dinámica familiar transfronteriza que ocurre en esta región y de la conformación de un espacio familiar transfronterizo. En este apartado las familias transfronterizas son construidas desde las relaciones de parentesco de los trabajadores entrevistados y cuyos miembros de la familia pueden ubicarse tanto en territorio guatemalteco como mexicano. Es de particular interés conocer las prácticas transfronterizas de las familias para llevar a cabo el trabajo doméstico, el de cuidados y el trabajo remunerado necesarios para su reproducción social.

La principal hipótesis de trabajo es que las familias guatemaltecas, en las que al menos uno de sus integrantes realiza trabajo remunerado, es decir, trabajo migratorio, de forma permanente en Chiapas con movilidades de commuting, temporal o regresos esporádicos, lleva a generar un espacio social de reproducción cotidiana transfronteriza, es decir, que al menos una de sus actividades necesarias para la reproducción cotidiana se realizan en ambos lados de la frontera Guatemala-México de manera simultánea, lo que construye un espacio familiar transfronterizo.

\section{Características de la fuerza de trabajo guatemalteca migrante en el Soconusco, Chiapas}

De acuerdo a la Encuesta sobre Migración en la Frontera Sur de México (Emif Sur), por los dos puntos fronterizos de mayor flujo migratorio entre México y Guatemala -Tecún Umán y El Carmen, que colindan con Ciudad Hidalgo y Talismán-, en promedio se registraron 327 mil cruces al año de guatemaltecos que trabajaron en Chiapas en el período 2004-2015. ${ }^{10}$ Como es posible observar en la Gráfica 1, si bien existen ańos con mayor o menor flujo laboral transfronterizo, en la

\footnotetext{
${ }^{10} \mathrm{La}$ Emif Sur es una encuesta que mide movilidades o cruces fronterizos con motivos laborales, es decir, registra el flujo del movimiento de trabajadores guatemaltecos a Chiapas. Por esta razón, la encuesta no permite medir el número de personas ni de familias que participan en dicho flujo laboral transfronterizo.
} 
década de análisis el porcentaje de cruces de trabajadores commuters sistemáticamente representa alrededor de la mitad de estas movilidades. Los trabajadores commuters son aquellos que "cruzan cada día a trabajar del otro lado de la frontera y mantienen su lugar de residencia en Guatemala, es decir, a su país de origen. ${ }^{11}$

Gráfica 1. Cruces anuales de trabajadores migratorios guatemaltecos a Chiapas por tiempo de estancia, 2004-2015.

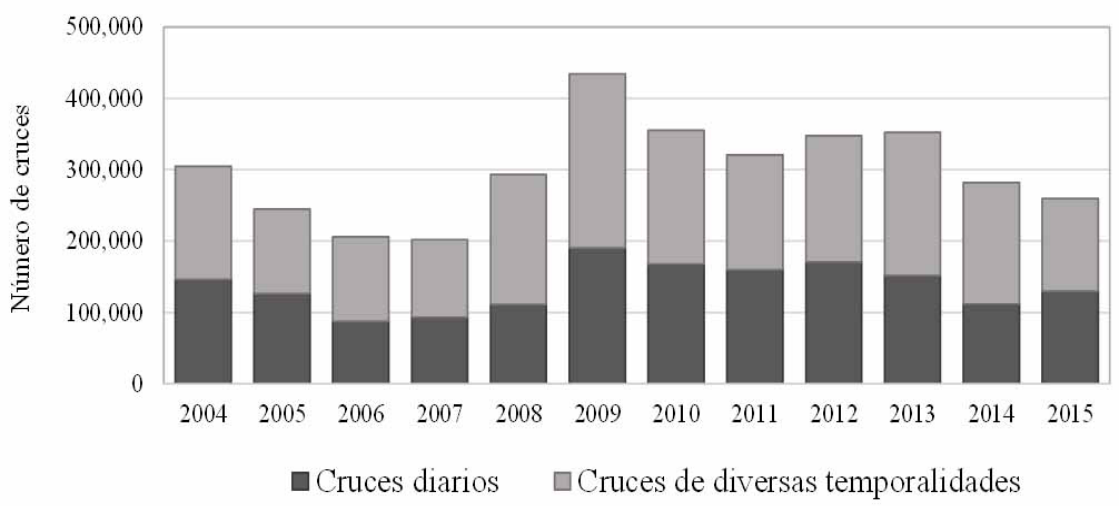

Fuente: Elaboración propia con base en Emif Sur 2004-2015. Flujo procedente de Guatemala a México por Tecún Umán y El Carmen

A continuación se presentan algunas de las principales características laborales y familiares del flujo anual de trabajadores migratorios transfronterizos a Chiapas en el año 2015, el cual se estimó en 246,041 cruces al año.

Es una fuerza de trabajo migrante que labora de manera cotidiana del otro lado de la frontera

En el año 2015, en el 99\% de los cruces registrados los trabajadores migratorios señalaron que su lugar de residencia habitual es Guatemala. El 51\% de los cruces fueron realizados por trabajadores commuters, es decir, guatemaltecos que cruzaron a laborar a Chiapas y regresaron a Guatemala el mismo día, mientras que en la otra mitad de los cruces laborales transfronterizos, los trabajadores guatemaltecos

\footnotetext{
${ }^{11}$ Los trabajadores commuters están definidos en ONU (1990), Artículo 2 (1).
} 
permanecieron en el Soconusco estancias de una semana (12\%), quince días (4\%), un mes (30\%) o más de un mes (3\%) en Chiapas y luego volvieron a Guatemala. Estos tiempos de estancia laborales son cíclicos ya que en más del $91 \%$ de los casos los trabajadores señalaron que volvería a Chiapas a trabajar, al otro día, una vez terminado el fin de semana o después de haber visitado a la familia algunos días.

La dinámica de movilidades y tiempos de estancia de la fuerza de trabajo guatemalteca en el Soconusco es habitual, se trabaja en territorio mexicano pero se mantiene el lugar de residencia habitual en Guatemala. Ante este panorama de alta y continua circularidad de trabajadores migratorios guatemaltecos en Chiapas resulta plausible considerar que el trabajo migratorio transfronterizo es una actividad relevante para los trabajadores y sus familiares el generar los recursos económicos necesarios para la manutención cotidiana.

Existe una región laboral transfronteriza claramente definida en la que se conjuga la demanda y oferta de fuerza de trabajo migrante

Como se observa en el Mapa 2, la dinámica regional transfronteriza ocurre y está enmarcada por algunos municipios guatemaltecos de donde provienen los trabajadores migratorios y algunos municipios mexicanos a los que se dirigen a trabajar. La región transfronteriza está delimitada por municipios muy cercanos a la línea fronteriza México-Guatemala y cuya amplitud espacial depende de si las movilidades transfronterizas son diarias o de commuting, o de mayores tiempos de estancia. En ambas movilidades se destaca que los trabajadores guatemaltecos residen y provienen del municipio fronterizo de Malacatán — departamento de San Marcos - y del municipio de Coatepeque - departamento de Quetzaltenango.

En el caso del flujo de trabajadores commuters el número de municipios guatemaltecos de procedencia es menor y son más cercanos a la línea fronteriza que en el flujo de diversas temporales, en el que hay un número más amplio de municipios y departamentos de Guatemala - como Retalhuleu, Suchitepéquez y Totonicapán-. En contraparte, el municipio de mayor recepción de trabajadores es Tapachula, tanto para los trabajadores commuters como los de otras temporalidades y 
destacan otros municipios más cercanos a la frontera como Suchiate y Ciudad Hidalgo.

La ubicación espacial de las movilidades laborales transfronterizas de guatemaltecos al Soconusco muestra que hay una zona claramente definida de la que sale los trabajadores - $95 \%$ proviene del Suroccidene de Guatemala - y los lugares a los que llegan — $-97 \%$ trabajadores en el Soconusco-. Estos referentes hacen plausible considerar que la región transfronteriza es el resultado de la conjugación de cortas distancias entre origen y destino laboral, además de las posibilidades del cruce y estancia en territorio mexicano y la demanda de fuerza de trabajo migrante en esta región ya indicados en la primera parte de este trabajo.

La demanda de fuerza de trabajo migrante guatemalteca es en todos los sectores de la economía del Soconusco

La estructura económica de la región del Soconusco está compuesta por una gran representación del sector agropecuario, particularmente agrícola, en el cual los empleadores - finqueros, ejidatarios y empresarios - demandan fuerza de trabajo de manera continua para llevar a cabo las actividades necesarias para mantener la producción de café, banano, cańa, papaya, mango, limón, entre otras. De acuerdo a los datos de la Emif Sur 2015, un tercio de los cruces de trabajadores guatemaltecos a la región fueron para emplearse en el sector agrícola -31.5\%—. Sin embargo, casi el 70\% participaba en sectores no agrícolas como el comercio al por menor y la industria de la construcción -23.8 y $19.5 \%$, respectivamente. 
Mapa 2. Municipios de origen y destino de la fuerza de trabajo migrante al Soconusco, según tiempo de estancia laboral en Chiapas (2015).
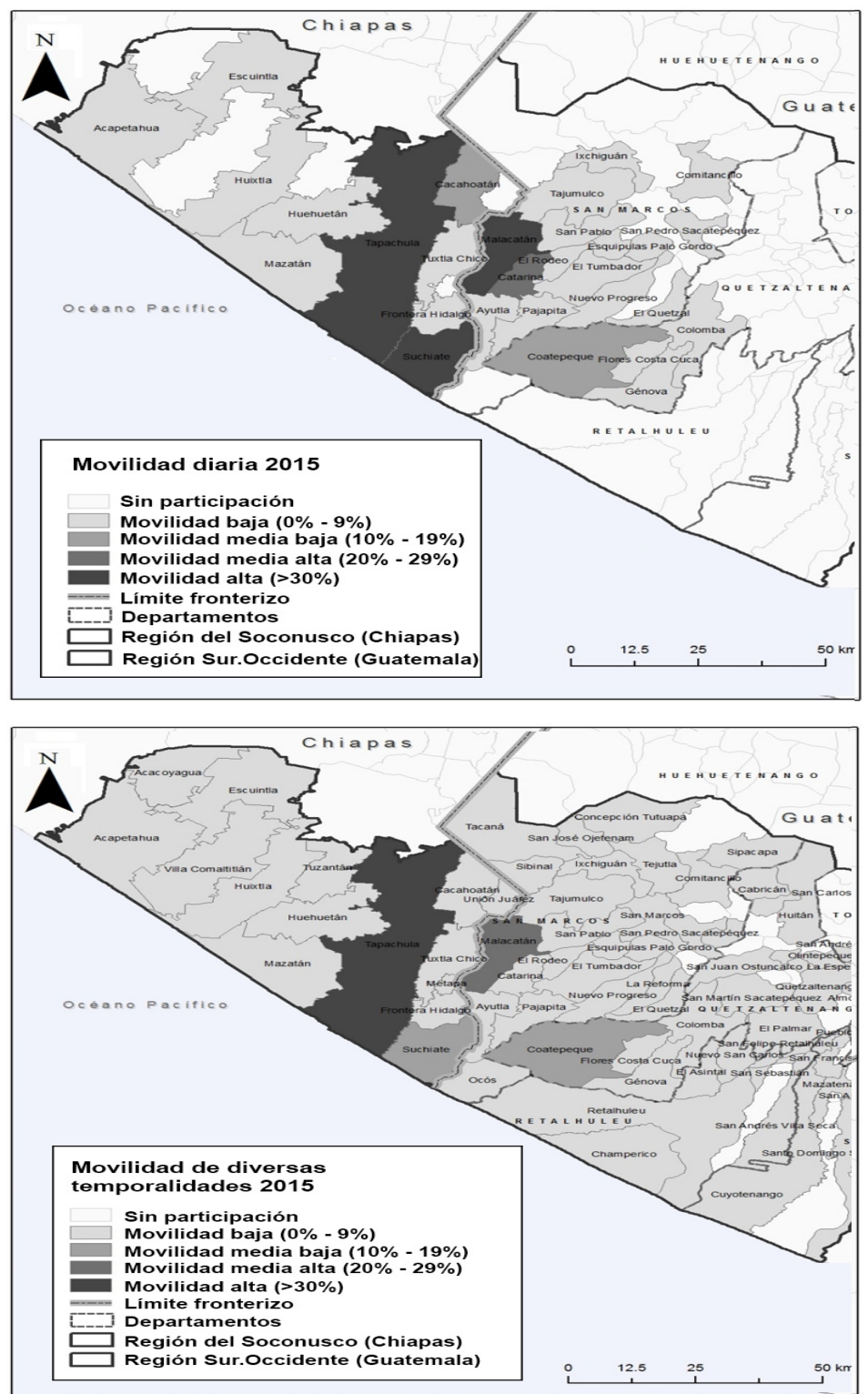

Fuente: Elaboración propia con base en Emif Sur 2015 
La Gráfica 2 permite mostrar que la contratación de fuerza de trabajo guatemalteca ocurre en todos los sectores de la economía del Soconusco y presenta sustantivas diferencias si se observa su distribución entre las movilidades laborales de commuters y las movilidades temporales. El sector de la construcción, los servicios y el trabajo doméstico en los hogares son los nichos laborales para la contratación de trabajadores commuters —29, 16 y 10\%, respectivamente—; así como el caso del comercio al por menor para los trabajadores guatemaltecos sin vínculo laboral con un empleador - 36\%-. En el caso de las estancias temporales, la contratación se concentra en el sector agrícola - 58\%- y en menor medida en hogares para realizar trabajo doméstico, el comercio al por menor y en la construcción - 12, 11 y 10\% respectivamente-. Estos resultados permiten mostrar la heterogeneidad de sectores de la economía en los que se demanda y contrata a fuerza de trabajo migrante en el Soconusco, es decir, la diversidad laboral mostrada en varias investigaciones (Ordoñez, 1993; Nájera, 2009, 2014).

Gráfica 2. Sector de actividad donde laboran los trabajadores migrantes guatemaltecos en Chiapas, según tiempo de estancia (2015).

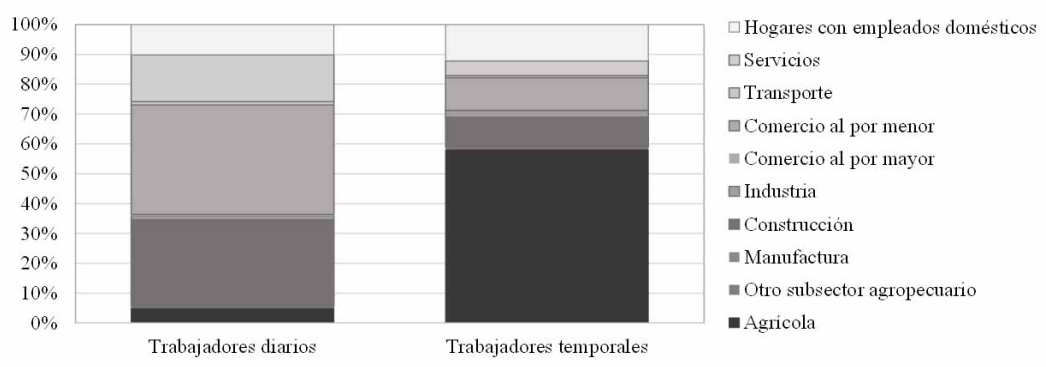

Fuente: Elaboración propia con base en Emif Sur 2015

Una particularidad entonces del Soconusco, como destino laboral, es que los trabajadores migratorios guatemaltecos llegan a zonas rurales y urbanas. ${ }^{12} \mathrm{El}$ tipo de sector económico al que se incorporan

${ }^{12} \mathrm{El} 71.9 \%$ de los cruces son a ciudades o pueblos, especialmente cercanos a la línea fronteriza Guatemala-México, como Tapachula, Suchiate, Cacahoatán, Tuxtla Chico, Unión Juárez y Metapa, que son espacios residenciales y comerciales. El menor número de movilidades laborales transfronterizas ocurren a espacios rurales, como fincas, ejidos o ranchos $(28.1 \%)$, hacia municipios fronterizos como Tapachula, Suchiate, Cacahoatán y Unión Juárez, y 
los trabajadores migratorios guatemaltecos, así como los lugares en donde laboran muestra la existencia de un mercado laboral mixto. ${ }^{13}$ La diversidad de sectores económicos de contratación de trabajadores migratorios en el Soconusco muestra la fuerte interacción entre la demanda de mano de obra y la disponibilidad de la población guatemalteca en trasladarse a diversos destinos laborales, más cerca o más lejos, rurales o urbanos, para trabajar en el Soconusco.

\section{Una oferta de fuerza de trabajo migrante sociodemográficamente diversa y con distintas posibilidades de movilidad laboral transfronteriza}

La mayoría de los cruces de trabajadores migratorios guatemaltecos a Chiapas son realizados por varones y sólo un $23.6 \%$ por mujeres. Sin embargo, las mujeres trabajadoras migratorias participan más en los cruces diarios o como commuting que en otras temporalidades - 27.9 y $19.2 \%$ respectivamente- y el estado civil también delimita el tiempo de estancia, ya que las mujeres casadas o unidas realizan preferentemente movilidades diarias $(74.7 \%)$ como se muestra en la Gráfica 3. En el caso de las movilidades laborales transfronterizas de varones, sean jefes de hogar o hijos, y de las mujeres hijas, participan en todos los tiempos de estancia, y son una subpoblación que permanece mayores tiempos de estancia laboral en Chiapas que las mujeres esposas.

De manera especial, hay que destacar que el $46.67 \%$ de los cruces de trabajadores migratorios está integrado por varones jefes de hogar, los cuales indicaron ser el principal proveedor económico en sus hogares (97.4\%); mientras que únicamente el $4 \%$ de los cruces realizados por trabajadoras esposas, hijos e hijas indicaron ser los responsables de dicha tarea en sus familias. La participación de esposas, hijas e hijos, muestra que en algunas familias guatemaltecas existen formas de proveeduría compartida en el hogar. Finalmente, aunque en la mayoría de las movilidades laborales transfronterizas los trabajadores guatemaltecos declararon movilizarse sin compañía a Chiapas $(94.4 \%)$,

municipios alejados de la línea fronteriza, como Mazatán, Huixtla, Escuintla, Huehuetán, Villa Comaltitlán, Tuzantán, Acacoyagua y Acapetahua.

${ }^{13}$ Es de destacar que las menores movilidades a zonas rurales no significan necesariamente una menor demanda de trabajadores migratorios transfronterizos, sino un mayor tiempo de estancia en México y por ello una menor movilidad entre Guatemala y México. 
en los traslados realizados por mujeres casadas en estancias temporales, el $35.9 \%$ viajó acompañada por sus hijos.

Grafica 3. Distribución de los cruces anuales de trabajadores migratorios transfronterizos de guatemaltecos a Chiapas, según tiempo de estancia en México y posición en la estructura familiar (2015)

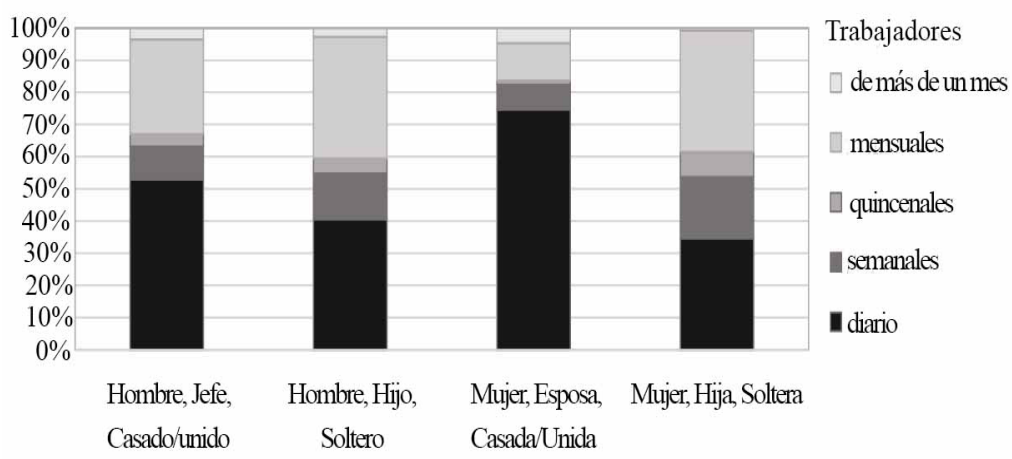

Fuente: Elaboración propia con base en Emif Sur 2015

Este panorama laboral, migratorio y familiar del flujo de trabajadores migratorios en el Soconusco muestra que la dinámica y características de la fuerza de trabajo migratorio y del mercado laboral chiapaneco son elementos que contribuyen a comprender la conformación de un espacio socio laboral transfronterizo, con una dinámica específica y propia; particularmente cuando, con base en investigaciones anteriores (Nájera, $2011,2014)$, se ha mostrado que este perfil migratorio y laboral es consistente desde su captación en la Emif Sur (2004) hasta la actualidad.

\section{Dinámica y reproducción social de las familias transfronterizas}

El contexto laboral transfronterizo entre el Soconusco y Guatemala hasta aquí presentado junto con el hecho de que los trabajadores indican mantener el lugar de residencia habitual en Guatemala, como el lugar donde está la casa y la familia, mientras ellos laboran en Chiapas, promueve dinámicas y prácticas transfronterizas necesarias para llevar a cabo la reproducción cotidiana de los propios trabajadores y sus familiares. En este sentido se parte de algunas hipótesis, que desde el interés 
en los estudios de familias, sirven de orientación para el análisis de las familias guatemaltecas entrevistadas:

a) la mayor presencia de trabajadores varones puede obedecer a que son los principales responsables de la manutención en su familia y la movilidad o migración laboral transfronteriza es la forma de acceder a un trabajo remunerado;

b) la presencia de mujeres trabajadoras migratorias esposas es un reflejo de las necesidades económicas de las familias guatemaltecas y seguramente se llevarán a cabo acciones para reorganizar la vida doméstica y de cuidados de los hijos en Guatemala;

c) el tiempo de estancia laboral en Chiapas, que puede definirse tanto por las necesidades de los empleadores como por las necesidades familiares de las y los trabajadores migratorios en determinada posición familiar, impacta en la organización y dinámica de la vida familiar transfronteriza.

A continuación se presentan algunas de esas prácticas sociales transfronterizas a partir del análisis de 40 entrevistas realizadas a trabajadoras y trabajadores guatemaltecos que laboraban en el Soconusco, como trabajadores commuters o en diversas temporalidades. ${ }^{14}$

Estructura, organización y prácticas transfronterizas de las familias guatemaltecas $\mathrm{Al}$ igual que en los datos de la Emif Sur, las y los guatemaltecos entrevistados en su mayoría indicaron vivir de manera habitual en Guatemala, por lo que su categoría de trabajadores migratorios refiere a una necesidad laboral solventada en Chiapas. Para la construcción de las familias estudiadas o de referencia, la relación de parentesco del trabajador con los integrantes de su familia fue el principal mecanismo para ubicar geográficamente a las familias, en las cuales algunos miembros vivían en Guatemala y otros en Chiapas.

\footnotetext{
${ }^{14}$ Las entrevistas fueron realizadas en el año 2012, en los municipios de Tapachula, Suchiate, Tuxtla Chico y Escuintla, y tuvieron como propósito general conocer las formas de organización intrafamiliar entre trabajadores guatemaltecos que laboraban en el Soconusco. Para una mayor información al respecto ver Nájera, 2014.
} 
En el análisis de las familias en situaciones de migración y dinámicas transfronterizas, el tiempo de estancia laboral en Chiapas resultó ser un elemento relevante para comprender la cotidianidad de las familias. El tiempo de la movilidad o migración transfronteriza es el tiempo de ausencia en el hogar en Guatemala. En el caso particular de las movilidades de commuting al tiempo de trabajo se le agrega el tiempo del traslado transfronterizo de la casa en Guatemala y al lugar de trabajo en Chiapas. En general, las ausencias de corto tiempo y en las que se moviliza únicamente el varón jefe de hogar, son las que menos cambios generan en la estructura y dinámica familiar; y por el contrario, las ausencias de mayor tiempo y en las que se moviliza la esposa o cónyuge son las que presentan los mayores cambios en la familia (cuadro 1).

Aunque la mayoría de los trabajadores señaló que mantienen su lugar de residencia habitual en Guatemala, en la cotidianidad la familia puede vivir dividida entre Guatemala y México, y en casos particulares, todos vivir en Guatemala - trabajadores commuters - o mantener la casa cerrada hasta que la familia completa regresa a Guatemala — trabajadores casi permanentes - . Por ello, los ajustes en la estructura familiar depende de quién o quienes se movilizan a territorio mexicano y del tiempo que permanecen trabajando $-y$ viviendo- del otro lado de la línea fronteriza.

Cuadro 1. Intensidad de cambios en la estructura y dinámica de las familias de trabajadores migratorios transfronterizos, Guatemala-Chiapas.

\begin{tabular}{|c|c|c|c|}
\hline \multirow{2}{*}{\multicolumn{2}{|c|}{$\begin{array}{l}\text { Tiempo de ausencia del(os) miembro(s) } \\
\text { de la familia }\end{array}$}} & \multicolumn{2}{|c|}{ Nivel de cambios familiares } \\
\hline & & en la estructura & en la dinámica \\
\hline \multirow{2}{*}{ Diario } & Jefe & Nulo & \\
\hline & Jefe y cónyuge & & \\
\hline \multirow{2}{*}{ Semanal } & Jefe & & \\
\hline & Jefe y cónyuge & & \\
\hline \multirow{3}{*}{$\begin{array}{l}\text { Mensual o } \\
\text { más tiempo }\end{array}$} & Jefe & & \\
\hline & Jefe y cónyuge & & \\
\hline & Familia incompleta & & Intenso \\
\hline
\end{tabular}

Fuente: Elaboración propia con base en entrevistas a trabajadores guatemaltecos en el Soconusco (2014)

Por ejemplo, en la familia mostrada en el Diagrama 1, cuando ambos cónyuges se movilizan a trabajar a Chiapas de manera casi permanente 
—estructura b y c_, y algún hijo se queda en Guatemala, ${ }^{15}$ éste se traslada al hogar de los abuelos y los padres viven en el espacio de trabajo - la finca, el mercado o la casa en la que trabajan- o en un cuarto rentado para dicho propósito, aunque sea "temporalmente". En estos casos, la familia "desaparece temporalmente" en Guatemala, ya que la casa suele permanecer cerrada hasta que los padres vuelven y todos los miembros de la familia se vuelven a reencontrar, lo que puede ocurrir cada fin de semana, cada quince días, cada mes o, en el caso más extremo, una vez al año tradicionalmente en Navidad o Semana Santa.

Diagrama 1. Estructuras de familias guatemaltecas con dinámicas laborales transfronterizas Guatemala-Chiapas.

a. Familia con trabajadora comnuter

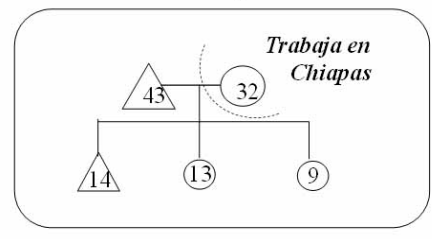

c. Familia con trabajadores estacionales (café)

Trabajan en Chiapas

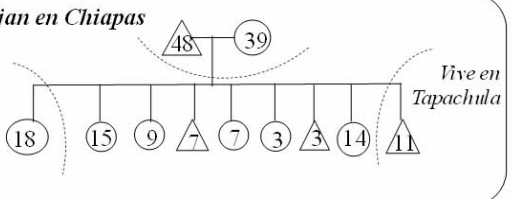

b. Familia con trabajadores semanales

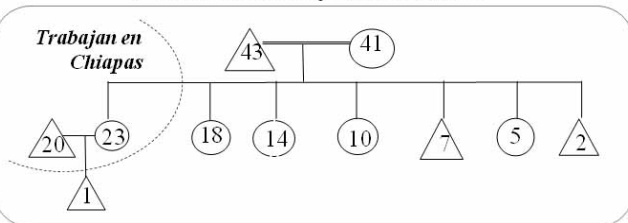

d. Familia con trabajadores temporales

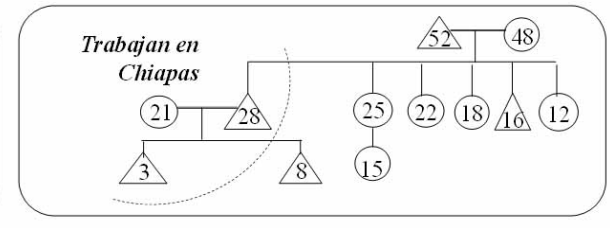

Fuente: Elaboración propia

En el caso de los ejemplos de familia presentados en las estructuras a y c del Diagrama 1, la estructura familiar no se modifica ante la salida de alguno o varios de los miembros de la familia por trabajar en Chiapas, ya que en el primer caso el o la trabajadora transfronteriza sale cada mañana a México pero vuelve cada tarde a Guatemala — trabajador

${ }^{15}$ Se privilegia que los hijos se queden en Guatemala porque se considera en general que "están mejor allä" porque tienen pleno acceso al sistema de educativo y de salud, es su lugar de pertenencia y ahí se encuentra la casa, la familia y los amigos. Si bien, algunas madres guatemaltecas llevan a sus hijos a Chiapas mientras aún requieren de cuidados maternos, una vez que cumplen seis años, la edad obligatoria para ir a la escuela, los hijos se trasladan a Guatemala. 
commuter-, y en el segundo caso, la ausencia de algunos integrantes de la familia es solo durante una época o temporada específica del año - períodos desde 1 a 3 meses_, como la época de cosecha de café, por lo que el resto de los integrantes de la familia permanecen en el mismo hogar en Guatemala hasta que finaliza dicho período.

Los miembros de estas familias transfronterizas no consideran necesaria la corresidencia en un mismo lado de la frontera Guatemala-México para ser familia. Las y los trabajadores migratorios transfronterizos siguen siendo "parte de la familia" porque continúan asumiendo las responsabilidades que les corresponden en ella — aunque de formas distintas-. Las tareas domésticas y el cuidado de los hijos son responsabilidad de las cónyuges o esposas, lo que implica reacomodos en la organización cotidiana ante su ausencia por razones laborales, sea ésta por algunas horas, semanas, quincenas o meses. Dos acciones son las más comunes en estas familias, la primera es incorporar a alguien que "le ayude" a la madre a desempeñar dichas tareas en Guatemala, lo que afecta la estructura y la dinámica de la familia; y la segunda, es reorganizar el tiempo y quiénes desempeñan tales tareas a través de transferir las responsabilidades a otras mujeres, como las hijas, sobrinas, tías o abuelas, y en casos extremos, se pide el apoyo de alguna vecina o amiga.

Cabe enfatizar que el caso más extremo de cambios en la estructura y dinámica de las familias transfronterizas analizadas fue el de las trabajadoras migratorias cónyuges que laboran casi de manera permanente en Chiapas y que llevan consigo a los hijos pequeños y dejan a otros hijos en Guatemala ${ }^{16}$. En estas familias transfronterizas, los hijos que se quedan en Guatemala suelen trasladarse al hogar de los abuelos donde otras mujeres se harán cargo de las labores de cuidados y las tareas domésticas que se requieran; mientras que en Chiapas la familia nuclear también lleva a cabo sus propias tareas domésticas, de cuidados y de trabajo remunerado pero en territorio mexicano. Es así que la familia nuclear "desaparece temporalmente" en Guatemala y “aparece temporalmente” en México.

${ }^{16}$ Una práctica común en las familias guatemaltecas entrevistadas es dejar en Guatemala a los hijos que están en edad escolar con el propósito de asegurar el acceso a la educación, y por lo tanto, llevar a Chiapas únicamente a los hijos que aún no están en edad de incorporarse al sistema educativo. 
Respecto a las formas de convivencia familiar, los relatos de las y los trabajadores migratorios entrevistados permitieron identificar que las prácticas familiares transfronterizas más comunes son los regresos cotidianos a Guatemala, las llamadas telefónicas, el envío de dinero y regalos, y llevar a los hijos a Chiapas durante el período vacacional en Guatemala. El propósito de estas prácticas transfronterizas es mantener el vínculo y mostrar el compromiso que se tiene con la familia, a pesar de estar separados en uno y otro lado de la frontera Guatemala-México.

Por su parte, mantener la relación conyugal y paterno-filial es una práctica profundamente valorada en estas familias, ya que se fortalece el compromiso y el cariño familiar. Aunque estas prácticas son de tipo bidireccional, tanto de los que se ausentan como de los que se quedan en Guatemala, son particularmente esperadas de los que se van a trabajar a Chiapas. De éstos últimos se espera que regresen a casa en las fechas pactadas, que lleven o envíen dinero para las necesidades familiares, y sobre todo, que tengan la disponibilidad para regresar a Guatemala ante una emergencia o necesidad familiar.

En estas dinámicas familiares transfronterizas también suelen haber desacuerdos, formas de control entre los cónyuges y de padres a hijos, e incluso, rupturas familiares. Las situaciones más comunes son el "acompañamiento forzoso" entre los jefes de hogar y las cónyuges como una forma de control; y la vigilancia transfronteriza a partir de las redes sociales, familiares y vecinales, las cuales funcionan en ambos lados de la frontera. Estas redes de información transfronterizas se caracterizan por tener un control social diferenciado por género y estado civil, en el que las mujeres y las personas casadas o unidas son los grupos de población más vigilados, en contraposición se encuentran los varones y las personas solteras como las menos vigiladas y sancionadas socialmente.

Es de destacar que la ausencia temporal de algún miembro de la familia por la búsqueda de un trabajo remunerado en Chiapas es el medio que les permita sostener, mantener y en ocasiones mejorar las condiciones de vida de ellos y sus familiares. Por ello, la ausencia del varón jefe del hogar, como el principal responsable de la manutención en la familia, es "más comprendida" por la familia que la ausencia de la cónyuge. Sin embargo, el trabajo migratorio transfronterizo tanto 
del jefe como de la cónyuge es visto como "un trabajo para la familia", que puede significar "un sacrificio" si los integrantes de la familia viven separados entre Guatemala y Chiapas, o un "beneficio" si la familia se mantiene junta en Guatemala - como ocurre con las familias de trabajadores commuters y trabajadores estacionales.

La vida cotidiana de las y los trabajadores guatemaltecos que componen la fuerza de trabajo migrante en el Soconusco no es posible reconocerla en toda su dimensión y consecuencias sino se incorpora la vida familiar. En este sentido, la salida temporal de uno o varios integrantes de las familias guatemaltecas promueve la reestructuración y reorganización de la vida familiar, no sólo en el lugar de origen o de residencia habitual sino también en los lugares de destino. Las familias guatemaltecas que participan en esta dinámica laboral transfronteriza se reorganizan, se dividen y, en casos extremos, se desintegran.

Finalmente, cabe señalar que las familias guatemaltecas de referencia son unidades sociales dinámicas que ajustan su estructura y prácticas cotidianas ante las necesidades, recursos y contexto en el que se ubican, y pueden transformarse, en cortos período de tiempo o de manera casi permanente de "familias transfronterizas" a "familias fronterizas" o incluso a "familias inmigrantes", en función de las posibilidades de satisfacer sus necesidades en uno o los dos lados de la línea fronteriza. En estas familias, el trabajo remunerado realizado en el otro lado de la frontera, entendido como trabajo migratorio transfronterizo, es el elemento vinculante y creador de "familias y espacios transfronterizos".

\section{Consideraciones finales}

Cuando miramos geográficos específicos de México reconocemos modalidades migratorias poco estudiadas como son las movilidades, migraciones y espacios transfronterizos; de la misma manera cuando miramos más allá de la dimensión laboral de las migraciones, encontramos que las y los trabajadores migratorios son padres, madres e hijos que reorganizan la vida familiar para mantenerse como grupo social. La frontera sur de México, y en particular la región del Soconusco y su contraparte la región fronteriza del suroccidente de Guatemala, existe un espacio con una estructura económica, política, demográfica y social 
relativamente "estable" en el tiempo, en la que de manera consistente el mercado laboral chiapaneco ha demandado fuerza de trabajo migrante que le permita llevar a cabo el desarrollo económico, productivo y social de la región. Algunas características laborales y migratorias del flujo de trabajadores transfronterizos guatemaltecos se han mantenido con el paso de los años y otras se han modificado; en el año 2015, con base en la encuesta EMIF SUR, continua evidenciándose que existe una demanda y contratación de fuerza de trabajo migrante guatemalteca en todos los sectores de la economía del Soconusco.

Al menos la mitad de los cruces de trabajadores migratorios al Soconusco son trabajadores commuters y el resto son trabajadores estacionales que permanecen entre uno y tres meses en Chiapas hasta volver nuevamente a Guatemala o de temporada, y trabajadores temporales con estancias casi permanentes en territorio mexicano. Todas estas formas de participación de los trabajadores migratorios en el mercado laboral del Soconusco han creado un espacio laboral transfronterizo moldeado por la propia demanda de mano de obra y las características de la oferta de trabajadores migratorios en la región.

La incorporación de la perspectiva sociodemográfica a un objeto de estudio tradicionalmente laboral permitió mostrar que los trabajadores migratorios no sólo son mano de obra migrante o fuerza de trabajo en la región del Soconusco, son también padres, madres, hijas e hijos, con vida familiar que debe llevarse a cabo a partir de prácticas transfronterizas, lo que les permite mantener su reproducción social. En esta mirada social, la relación de parentesco y el compromiso familiar se destacaron como elementos esenciales para identificar a las familias en este contexto transfronterizo, y para comprender las acciones de las y los trabajadores migratorios por su posición familiar — como el tiempo de estancia laboral en el Soconusco.

La ausencia temporal de uno o varios integrantes de la familia por razones laborales provoca cambios en la estructura y dinámica cotidiana de dichas unidades sociales, generalmente ubicadas en Guatemala; estos cambios pueden ser menores, como en el caso de las dinámicas transfronterizas de commuting, o mayores como en el caso de las familias en las que los padres — jefe de hogar y cónyuge_ laboran 
casi de manera permanente en el Soconusco. Sin embargo aunque en las familias con movilidades laborales de commuting no se observaron cambios en la estructura familiar, en todas las familias se observaron ajustes en la dinámica intrafamiliar para llevar a cabo las tareas necesarias para la reproducción cotidiana, como el trabajo doméstico, el trabajo de cuidados y el trabajo extradoméstico remunerado.

El acercamiento cualitativo a los trabajadores y sus familiares permitió mostrar la relevancia del trabajo remunerado transfronterizo o trabajo migratorio como medio para asegurar la reproducción social de las familias de referencia. La búsqueda de un trabajo remunerado para adquirir los bienes y servicios necesarios en la familia lleva a los principales responsables de dicha tarea, generalmente los varones jefes de hogar, a trasladarse "a donde haya trabajo" — como bien muestra la Emif Sur-. Por otra parte, se visibilizó la relevancia del trabajo remunerado de las cónyuges en la manutención de la familia, y en especial la existencia de movilidades laborales transfronterizas "en pareja”, en modelos de proveeduría conyugal compartida y en experiencias migratorias en las que los hijos suelen quedarse en Guatemala.

Sin embargo, la fortaleza de una tradicional división del trabajo intrafamiliar en las familias guatemaltecas estudiadas significa que las actividades asociadas a varones y mujeres "se llevan a cualquier lado de la frontera"; en este sentido, las cónyuges que son parte de la fuerza de trabajo migrante en el Soconusco incrementan su trabajo cotidiano total y desarrollan estrategias transfronterizas para asumir las responsabilidades domésticas y de cuidados de sus hijos, independientemente del lado de la línea fronteriza Guatemala-México en la que se encuentren.

Para las familias guatemaltecas de referencia, la vida cotidiana se organiza a partir del trabajo remunerado transfronterizo, por lo que las condiciones de laborales en Chiapas se convierten en una dimensión relevante. En este sentido, trabajar para un patrón o por cuenta propia, en un horario de trabajo fijo o movible, y en espacios alejados o cercanos a la línea fronteriza, o en los que se pueda tener o no a la familia $-\mathrm{y}$ en particular a hijos pequeños_ son elementos relevantes para moldear la vida familiar en este espacio con dinámicas laborales transfronterizas. 
La mirada sociodemográfica en el conocimiento de los trabajadores migratorios en el Soconusco permitió reconocer la existencia de "familias transfronterizas", es decir, familias en las que al menos una de las tareas necesarias para su reproducción social se realiza de manera cotidiana "del otro lado de la frontera". Si bien este trabajo tomó como eje de referencia el trabajo remunerado transfronterizo o trabajo migratorio, se mostró la existencia de otros trabajos transfronterizos, como el trabajo de cuidados y el trabajo doméstico, que en algunos casos también se realizan de manera simultánea en ambos lados de la línea fronteriza Guatemala-México, lo que contribuye a configurar espacios de vida transfronterizos.

El estudio de las familias en dinámicas transfronterizas permitió visibilizar retos para su identificación, definición y entendimiento debido a lo relativo que se vuelven conceptos como el lugar de residencia habitual y el significado del tiempo de estancia laboral para las familias y poblaciones en movimiento. Cabe señalar que aunque en este trabajo no se abordaron otras formas de residencia habitual en situaciones de movilidad y migración laboral transfronteriza, existen "hogares no familiares" en el Soconusco conformados por trabajadores guatemaltecos que comparten la organización de la vida cotidiana con personas sin lazos de parentesco; por lo que aún es posible seguir profundizando en las diversas formas en que las poblaciones en movimiento llevan a cabo y organizan la vida cotidiana.

Finalmente, cabe señalar que la histórica y continua demanda de fuerza de trabajo migrante en el mercado laboral del Soconusco ha creado un espacio laboral transfronterizo, que puede ser claramente identificado por la concentración de relaciones laborales entre determinados municipios del Soconusco y de la región del Suroccidente de Guatemala, y que dicho espacio laboralse le puede incorporar el espacio familiar y el espacio social transfronterizo que crean los trabajadores migratorios a partir de sus prácticas a través de la frontera para llevar a cabo su reproducción social y generacional. Este espacio transfronterizo entre Guatemala y México al parecer continuará en el futuro debido a las necesidades laborales de ambos lados de la línea fronteriza, por lo que el reconocimiento de un espacio que traspasa la frontera para la 
reproducción social de las familias guatemaltecas permite incorporar de la misma forma la reproducción social de las familias del Soconusco, mexicanas o extranjeras, que emplean a trabajadores migratorios en sus casas, negocios, fincas y ejidos, probablemente de manera permanente.

\section{Bibliografía citada}

Acosta, Felix, 2003, "La familia en los estudios de población en América Latina: estado del conocimiento y necesidades de investigación”, Papeles de Población, vol. 9, núm. 37, julio-septiembre, pp. 5-50. Acuña, G. Beatriz, 1980, "Transmigración legal en la frontera MéxicoEstados Unidos”, Revista Mexicana de Sociología, núm. 4, pp. 277-322.

Alegría, Tito, 1989, "La ciudad y los procesos transfronterizos entre México y Estados Unidos", Frontera Norte, vol. 1, núm. 2, pp. 53-90.

Arriagada, Irma, 2007, “Transformaciones familiares y políticas de bienestar en América Latina", en Familias y políticas públicas en América Latina: una historia de encuentros y desencuentros, Cepal, Naciones Unidas, Santiago de Chile, pp. 125-152.

Arriagada, Irma, 2012, Diversidad y desigualdad de las familias latinoamericanas. Desafios para las politicas públicas, Saarbrücken Alemania, Académica española.

Encuesta sobre Migración en la Frontera Sur de México 2010. Bases de datos: Flujo de Procedentes de México a Guatemala, Colegio de la Frontera Norte, Instituto Nacional de Migración, Consejo Nacional de Población, Secretaría del Trabajo y Previsión Social y Secretaría de Relaciones Exteriores, 2004-2015, STPS, SRE, Segob, INM, Colef, Conapo, México, disponible en https://www. colef.mx/emif/bases.php [consultado en línea el 6 de mayo de 2016].

Fauné, María Angélica, 1996, “Transformaciones en las familias centroamericanas. Agudización de la situación de inequidades de las mujeres", Serie Estudios Básicos de Derechos Humanos, vol. 4, pp. 1-53. 
García Guzmán, Brígida y Orlandina de Oliveira, 2006, Las familias en el México metropolitano: visiones femeninas y masculinas, El Colegio de México, México.

García Guzmán, Brígida y Orlandina de Oliveira, 2011, “Cambios familiares y políticas públicas en América Latina”, Annual Review of Sociology, núm. 37, pp. 613-633.

Godoy, H. y L. F. Lira, 1973, Aspectos sociológicos de la familia, Instituto de Sociología, Universidad Católica de Chile, Santiago de Chile.

Nájera Aguirre, Jéssica N., 2009, “Diversificación laboral y autoempleo entre los trabajadores migratorios guatemaltecos en Chiapas, México", en Jesús Arroyo y Salvador Berumen (coordinadores), Migración a Estados Unidos: remesas, autoempleo e informalidad laboral, Universidad de Guadalajara, Centro de Estudios Migratorios Segob y DGE Ediciones, México, D.F., pp. 279-314.

Nájera Aguirre, Jéssica N., 2011, "Formas de movilidad laboral transfronteriza de las y los guatemaltecos a Chiapas, una visión desde la familia”, Revista Latinoamericana de Estudios de la Familia, vol. 3, pp. 177-198.

Nájera Aguirre, Jéssica N., 2014, Movilidad laboral transfronteriza y vida familiar de los trabajadores guatemaltecos en Chiapas, México, Tesis de doctorado en Estudios de Población, CEDUA El Colegio de México, México, D.F, septiembre de 2014.

Oliveira, Orlandina de, 1989, "Empleo femenino en México, en tiempos de recesión económica: tendencias recientes”, en Cooper, Jennifer, Teresita De Barbieri, Teresa Rendón, Estela Suárez, Esperanza Tunón (compiladoras), Fuerza de trabajo femenina urbana en México, Coordinación de Humanidades UNAM, Miguel Ángel Porrúa, México, pp. 29-66.

Oliveira, Orlandina de y Vania Salles, 1989, "Acerca del estudio de los grupos domésticos: un enfoque sociodemográfico”, en Orlandina de Oliveira, Marielle Pepin Lehalleur y Vania Salles (compiladoras), Grupos domésticos y reproducción cotidiana, UNAM, Miguel Ángel Porrúa, El Colegio de México, México, pp. 11-37. Ordóñez Morales, César Eduardo, 1993, Eslabones de frontera, un análisis sobre aspectos del desarrollo agrícola y migración de fuerza de 
trabajo en regiones fronterizas de Chiapas y Guatemala, Universidad Autónoma de Chiapas, Chiapas.

Organización Naciones Unidas (ONU), 1990, Convención internacional sobre la protección de los derechos de todos los trabajadores migratorios y de sus familiares, Adoptada por la Asamblea General, Suiza.

Palma, Silvia Irene, 2003, Caracterización de los movimientos de la población en la frontera Guatemala-México, Flacso Guatemala, Guatemala.

Pedone, Claudia, 2006, “'Lo de migrar me lo tomaría con calma': representaciones sociales de jóvenes en torno al proyecto migratorio familiar", en Andrés Pedreño (coordinador), Tránsitos migratorios: Contextos transnacionales y proyectos familiares en las migraciones actuales, Universidad de Murcia, España, pp. 141-170.

Programa de las Naciones Unidas para el Desarrollo (PNUD), 2011, Cifras para el desarrollo humano San Marcos, Colección estadística departamental, núm. 12, PNUD Guatemala, Guatemala.

Rivera Farfán, Carolina (coordinadora), 2014, Trabajo y vida cotidiana de centroamericanos en la frontera suroccidental de México, Publicaciones de la Casa Chata, CIESAS, México.

Salles, Vania, 1991, "Cuando hablamos de familia, ¿de qué familia estamos hablando?”, Nueva Antropología, vol. 11, núm. 39, pp. 53-86.

Salvia, Agustín, 1995, "La familia y los desafíos de su objetivación: enfoques y conceptos", Estudios Sociológicos, vol. 13, núm. 37, pp. 143-162.

Woo Morales, Ofelia, 2006, “Transiciones familiares en la experiencia migratoria hacia Estados Unidos. El Caso de la zona metropolitana de Guadalajara”, ponencia presentada en el Segundo Coloquio Internacional sobre Migración y Desarrollo: Migración, Transnacionalismo y Transformación social, Hotel Hacienda Cocoyoc, Morelos, 26 a 28 de octubre.

Zebadúa, Emilio, 1999, Chiapas, historia breve, Secretaría de Educación Pública, El Colegio de México, México. 